\title{
A Light Light-source For Nasal Endoscopy Audit: The Ubiquitous Mobile Phone
}

Catherine Chapple, Ivor Kwame, Ryian Mohammed, Francis Vaz, Alam Hannan

\section{Background}

Fibre-optic nasal endoscopy (FNE) provides assessment of the nose and airway. We audited the practicality of standard wired light-boxes and portable hand-held light sources to our novel use of mobile phone flashlights to attend emergencies.

\section{Objectives}

Audit of the mobile phone flashlight feature as an adequate light source for FNE during emergent reviews.

\section{Methods}

We carried out thirty consecutive reviews of patients in casualty and on wards:

- The first ten using a commercially available portable light source (fig 1).

- The second ten were with a trolley-mounted wired light box (fig 2).

- The final ten using a mobile phone with light source connector held perpendicular to the flashlight on full beam (Fig 3 ).

We compared the respective portability, reliability (light quality) and availability (within 1 minute of obtaining FNE), scoring either 1 or 0 for satisfactory on each clinical encounter.

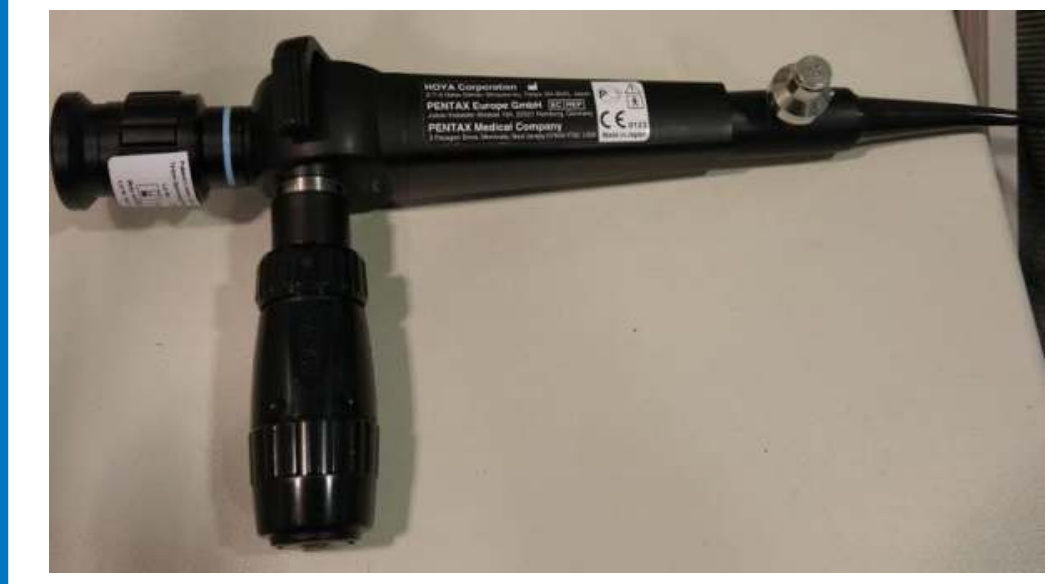
portable Pentax

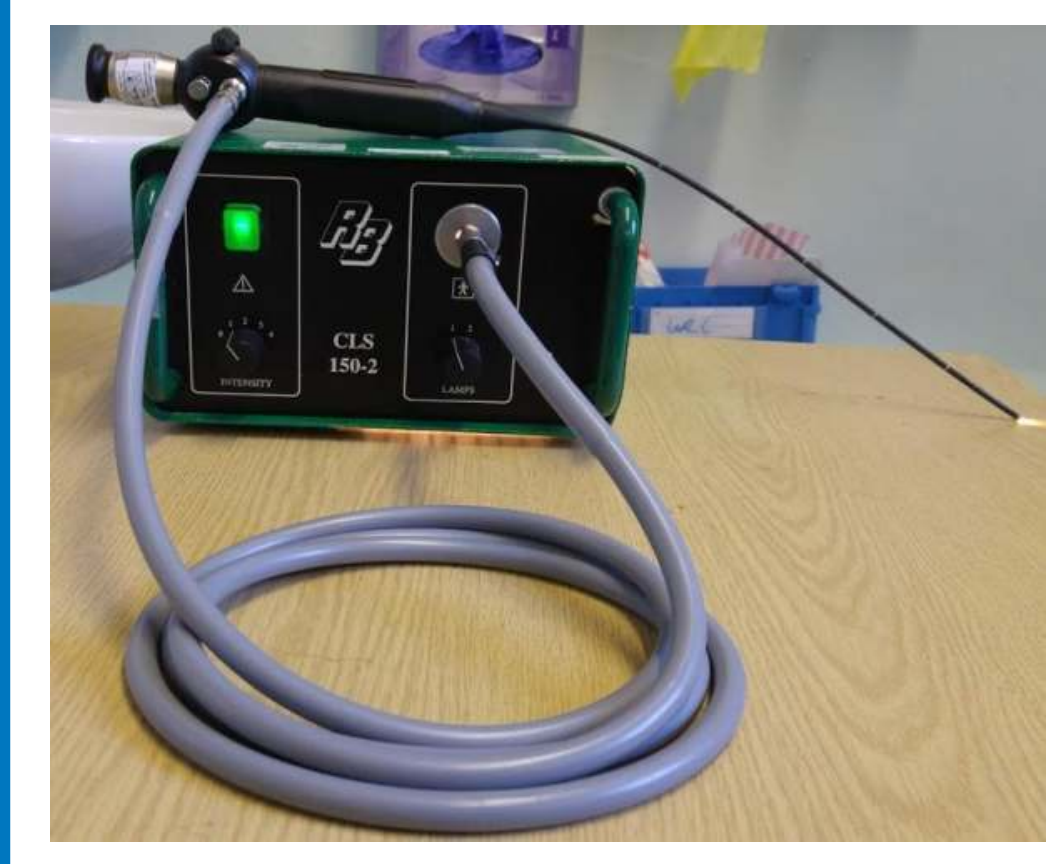

\section{Methods (cont.)}

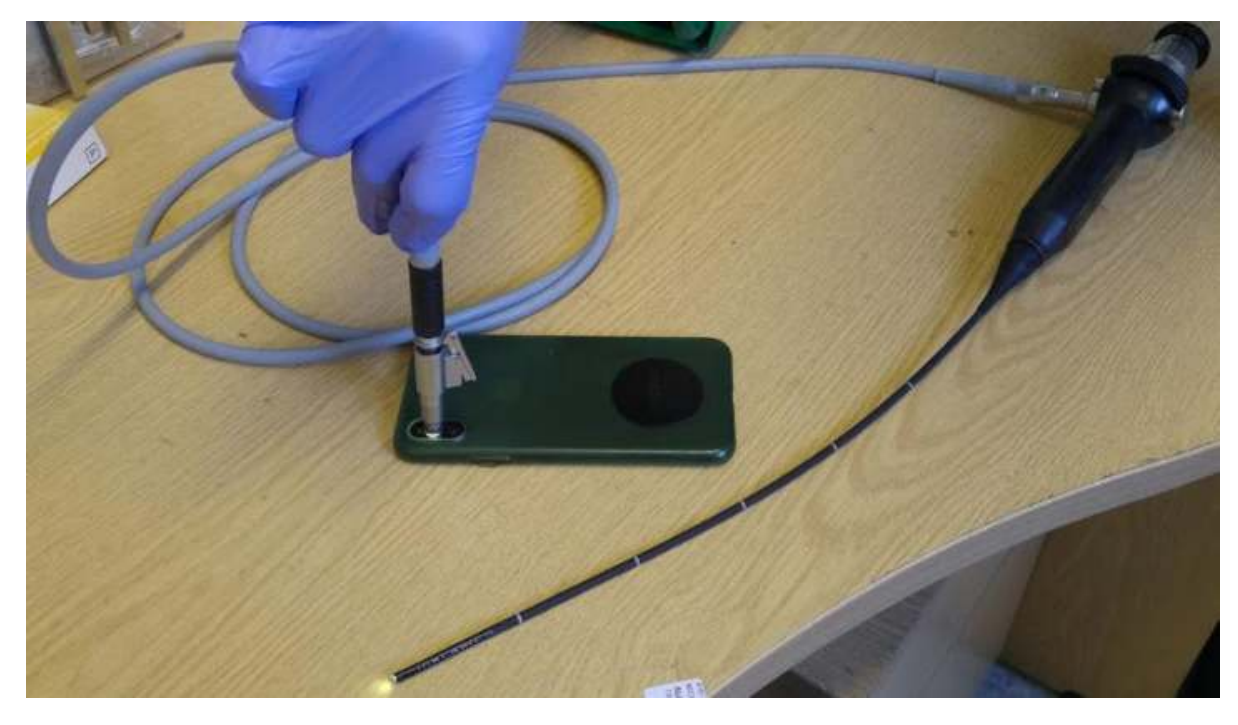

Figure 3. Fibre-

optic nasal endoscope connected to the mobile phone flashlight by holding the connector perpendicular to flashlight.

\section{Results}

Our results (summarised below) showed:

- The wired lightboxes were reliable (i.e. satisfactory light quality) but not readily portable or available.

- The hand-held light sources were reliable and portable, but often not readily available.

- Mobile phone flashlights were reliable, portable and readily available - but required an assistant to maintain connection.

Throughout the audit period it was never necessary to change from a mobile phone light source to either of the standard light sources.

Figure 1. Fibreoptic nasal endoscope with hand-held light source attached.

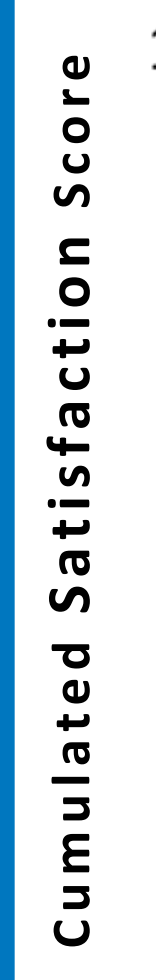

Figure 2. Fibreoptic nasal endoscope attached to a standard wired light-box.

\section{Conclusions}

Mobile flashlights were a reliable and available light source for FNE. Its ubiquitous nature facilitates speedy reviews, particularly advantageous in emergent settings - but requires an assistant. 